\title{
The Unsatisfied Patient after Cataract Surgery Ocular Surface Disease as a Major Contributor!
}

\author{
Adeeb M Rushdi Ubeid* \\ Department of Ophthalmology, Erbil Teaching Hospital, Erbil, Iraq
}

*Corresponding author: Adeeb M Rushdi Ubeid, Department of Ophthalmology, Erbil Teaching Hospital, Erbil, Iraq

\begin{abstract}
In the era of advanced technology in managing patients with cataracts, yet a number of patients remain unhappy. We daily face unsatisfied patients despite having 20/20 vision.

Many of the ocular symptoms are in the form of ocular irritation, stinging, and blurred vision. Unrecognized dry eye could be a major contributor to these symptoms.

Although cataract surgery is an intraocular surgery, it causes microscopic ocular surface damage that could worsen the preexisting ocular surface disease or leads to dry eye.

The aim of this article is to emphasis on the importance of assessing the state of ocular surface both subjectively and objectively and treat any critical issue before and after surgery to have a satisfied patient postoperatively.
\end{abstract}

\section{Keywords}

Cataract, Dry eye, Ocular surface disease

\section{Introduction}

According to P.H.C.H.O study, the incidence of dry eye in patients scheduled to undergo cataract surgery in a real-world setting is higher than anticipated.

In this observational study, it is found that about $77 \%$ of the eyes have positive corneal staining, $60 \%$ have tear film break up time of $\leq 5$ seconds, and $21 \%$ have Schirmer's scores of $\leq 5 \mathrm{~mm}$. The majority of patients didn't report symptoms that are suggestive of dry eye [1].

In addition to preexisting dry eye, cataract surgery evokes the ocular surface or makes asymptomatic dry eye symptomatic. The etiology is multifactorial and related to corneal nerves dissection, surface epithelium disruption and inflammation. This is usually evident postoperatively by ocular discomfort and fluctuation of vision.

The aim of this article is to review the current perspectives in dealing with ocular surface disease in the setting of cataract surgery and increasing patient satisfaction postoperatively.

\section{Assessment of Symptoms and Signs Related to Ocular Surface Disease (OSD)}

The presence of healthy ocular surface prior to cataract surgery is not only crucial for post-operative patient comfort, but also affects the IOL calculation and postoperative visual function.

Prior to surgery, a complete history and ocular examination, combined with the essential clinical tests, should be performed and documented for cataract patients for whom there is even a mild suspicion of dry eye disease (DED). Inquiry should about symptoms such as ocular burning, irritation, redness, tearing, artificial tear use, and visual fluctuations [2].

A history of systemic collagen disease, vascular disease, or associated manifestations such as arthritis or dry mouth provides important clues for the possibility of concomitant OSD [3].

Slit lamp examination is performed mainly to assess the state of ocular surface signs that are consistent with an abnormal surface including low meniscus tear height, lid margin abnormalities, evidence of meibomian gland dysfunction and a low tear film break up time (TBUT). The most important component of the slit lamp examination is dye staining of the cornea and conjunctiva. Fluorescein and/or lissamine green dye reveals punc- 
tate corneal and conjunctival staining in early moderate to severe DED. A Schirmer test of $5 \mathrm{~mm}$ or less is generally accepted as indicator of DED.

\section{New Objective and Evidence Based Testing}

There are new noninvasive tests to assess dye eye disease, these include tear osmolarity analysis (TearLab Osmolarity System; TearLab), detection of tear matrix metalloproteinase 9 (InflammaDry; Rapid Pathogen Screening), lipid layer interferometry (Lipiview; TearScience), wavefront aberrometry, and noncontact topography-based TBUT (Keratography 5; Oculus Optikgeräte). These tests will add value in diagnosing OSD/ DED more accurately.

\section{Ocular Surface Preparation Prior to Surgery}

A step wise and systematic approach is essential in managing OSD. Ocular surface preparation is beneficial not only in patients with established ocular surface disease, but also in those with minimal signs or symptoms of surface disease [4].

The primary therapy for mild dry eye will start with tear substitutes, this would improve the ocular signs and symptoms in many patients.

The likelihood of treatment beyond tears increases for moderate dry eye. Reducing inflammation of the ocular surface is a primary goal in the treatment of early moderate to advanced DED. A commonly prescribed regimen for cataract patients with moderate DED includes cyclosporine ophthalmic emulsion $0.05 \%$ (Restasis; Allergan, Inc.) dosed twice daily or lifitegrast in conjunction with a tapering course of a topical steroid.

An important consideration in patients with cataract surgery is to maximally suppress inflammation preoperatively, given that inflammation increases significantly after surgery.

In patients with significant aqueous deficiency, consideration should be given to punctual occlusion. Punctal plugs have been shown to improve vision in dry eye patients by stabilizing tear film through decreasing its osmolarity [5]. Combining punctual plugs with $0.05 \% \mathrm{cy}$ closporine is shown to result in the best Schirmer test scores, Rose Bengal staining, and reduction in overall artificial tear use compared to either treatments alone [6].

In patients with meibomian gland dysfunction (MGD) and blepharitis, hot compresses, lid hygiene, topical azithromycin, and oral omega-3 fatty acid supplements have demonstrated effective reduction of ocular surface morbidity. If OSD goes untreated before cataract surgery, it is likely to result in patient dissatisfaction and, although rarely, may be implicated in postoperative infections [7].

Newer treatments such as thermal pulsation systems (LipiFlow System; TearScience, Inc.), meibomian gland probes, and intense pulsed-light therapy may also be useful adjuncts for treating MGD in cataract patients.

Although OSD optimization will lead to a delay in cataract surgery but ensure better surgical predictability and outcomes. It is important to make patients aware of their diagnosis and treatment of ocular surface disease before surgery.

\section{Intra-operative Measures to Minimize Surface Damage}

A variety of factors in the operative period contribute to exacerbating symptoms. Frequent administration of preserved preoperative and postoperative eyedrops damage the epithelial surface [8].

The corneal incisions made at the time of surgery disrupt the feedback mechanism that stimulates the lacrimal gland by the corneal nerves. Prolonged exposure to the microscope's light, aggressive irrigation of the tear film and frequent topical anesthetic on the day of surgery all traumatize the corneal epithelium and/or cause conjunctival mucous goblet cell loss [9]. The use of light filters, decreased exposure time, proper irrigation, and gentle manipulation of the ocular surface tissue may reduce complications after surgery [10].

\section{Post-operative Ocular Surface Care}

The ocular surface typically gets worse after cataract surgery. Contributing factors include corneal nerve disruption, postoperative inflammation and prolonged use of preservative containing topical medications. In patients with surface disease it is preferred to stop or taper medications when no longer needed. Topical NSAIDs such as nepafenac, ketorolac and diclofenac have been reported to cause corneal melting mainly in the presence of epithelial breakdown [11,12]. Therefore, in patients with significant ocular surface disease, it is prudent to minimize or even avoid the use of topical NSAIDs especially as a single agent, usually in combination with topical steroids.

Increasing evidence suggests the efficacy of a combination of topical lubricants and topical cyclosporine for long-term ocular surface maintenance. Cyclosporine $0.05 \%$ therapy twice daily for 3 months has been reported to reduce dry eye signs and improve visual quality after cataract surgery. It is shown pre- and postoperative therapy with topical cyclosporine $0.05 \%$ helps alleviate dry eye signs, improve visual outcomes, and enhance the satisfaction of patients undergoing cataract surgery with multifocal IOL implantation [13].

\section{Conclusion}

Ocular surface disease frequently goes undiagnosed in the setting of cataract surgery specially when the patients are asymptomatic. Diagnosis and management of ocular surface disease in cataract patients will help to achieve better visual outcome and patient satisfaction postoperatively. 


\section{Disclosure}

The author has no financial disclosure to mention. No financial interest in any product mentioned.

\section{References}

1. Trattler WB, Majmudar PA, Donnenfeld ED, McDonald MB, Stonecipher KG, et al. (2017) The Prospective Health Assessment of Cataract Patients' Ocular Surface (PHACO) study: the effect of dry eye. Clin Ophthalmol 11: 1423-1430.

2. Edward C Lai, Christopher E Starr (2014) Managing Dry Eye Disease in Cataract Patients. Cataract \& Refractive Surgery Today.

3. Li XM, Hu L, Hu J, Wang W (2007) Clinical observation of dry eye in patients before and after cataract surgery. Zhonghua Yan Ke Za Zhi 43: 10-13.

4. Movahedan A, Djalilian AR (2012) Cataract surgery in the face of ocular surface disease. Curr Opin Ophthalmol 23: 68-72.

5. Gilbard JP, Rossi SR, Azar DT, Heyda KG (1989) Effect of punctal occlusion by Freeman silicone plug insertion on tear osmolarity in dry eye disorders. CLAO J 15: 216-218.

6. Roberts CW, Carniglia PE, Brazzo BG (2007) Comparison of topical cyclosporine, punctal occlusion, and a combination for the treatment of dry eye. Cornea 26: 805-809.

7. Sanghamitra Burman (2015) Cataract surgery and ocular surface disease. Cataract \& Refractive Surgery Today Europe.

8. Cha SH, Lee JS, Oum BS, Kim CD (2004) Corneal epithelial cellular dysfunction from benzalkonium chloride (BAC) in vitro. Clin Experiment Ophthalmol 32: 180-184.

9. Chuang J, Shih KC, Chan TC, Wan KH, Jhanji V, et al. (2017) Preoperative optimization of ocular surface disease before cataract surgery. J Cataract Refract Surg 43: 1596-1607.

10. Kasetsuwan N, Satitpitakul V, Changul T, Jariyakosol S (2013) Incidence and pattern of dry eye after cataract surgery. PLoS One 8: e78657.

11. Wolf EJ, Kleiman LZ, Schrier A (2007) Nepafenac-associated corneal melt. J Cataract Refract Surg 33: 1974-1975.

12. Khalifa YM, Mifflin MD (2011) Keratitis and corneal melt with ketorolac tromethamine after conductive keratoplasty. Cornea 30: 477-478.

13. Donnenfeld ED, Solomon R, Roberts CW, Wittpenn JR, McDonald MB, et al. (2010) Cyclosporine $0.05 \%$ to improve visual outcomes after multifocal intraocular lens implantation. J Cataract Refract Surg 36: 1095-1100. 